\title{
Pas de politique de santé sans la FMH
}

Une chose est claire: les gagnants de la votation du $1^{\mathrm{er}}$ juin, ce sont indubitablement les patientes et les patients. Jamais auparavant, en Suisse, une alliance aussi large ne s'était engagée avec autant de véhémence pour défendre leurs intérêts. Le personnel soignant, les dentistes, les pharmaciens, les associations de professionnels de la santé et les organisations de défense des patients et des consommateurs se sont tous battus aux côtés des médecins. Ensemble, nous avons mené une campagne intense et qui s'est soldée par un très grand succès. Par 69,5\% de non, le peuple suisse a massivement rejeté l'article constitutionnel «Qualité et efficacité économique dans l'assurance-maladie». Tous les cantons ont refusé cette modification de la Constitution, la Suisse romande encore plus nettement que la Suisse alémanique.

Ce résultat laisse peu de champ à l'interprétation: les Suisses et les Suissesses ne veulent pas d'expérimentations hasardeuses en matière de santé publique. Ce qu'ils veulent, c'est un système de santé équilibré et de haute qualité. Nous savons depuis la votation sur la caisse unique qu'ils souhaitent garder leurs caisses-maladie - et nous savons depuis le $1^{\text {er }}$ juin qu'ils les veulent à leur juste place!

Cela étant, personne ne conteste que le système de santé ait besoin de réformes. L'alliance qui a dirigé la campagne de vote sera appelée à jouer un rôle central dans leur réalisation. Elle devra profiter de son image nouvellement acquise d'acteur-clé pour imposer des aménagements qui soient dans l'intérêt des patientes et des patients.

La gestion du domaine de la santé par les pouvoirs publics, mais aussi le libre choix du médecin et la prise en charge des soins à domicile, font certainement partie des éléments essentiels d'un système équilibré et de qualité.
Mais il est clair qu'il y a là des niveaux de compétence différents, et le peuple l'a bien compris.

La régulation du système de santé, la définition de son cadre - fourniture des soins, démographie médicale, ... est une responsabilité politique qui appartient à l'autorité publique et à personne d'autre, notamment en aucun cas aux assureurs. Les médecins, par contre, doivent absolument être associés à ces procédures de décision.

Le fonctionnement du système ensuite, à l'intérieur du cadre fixé politiquement, doit lui être réglé entre «prestataires» et assureurs: tarifs, qualité, contrôle de la facturation ...

Ces niveaux de compétence différents ne doivent plus être confondus!

Après le $1^{\text {er }}$ juin, il convient maintenant de tirer parti de la dynamique acquise lors de la campagne pour élaborer des solutions, faire avancer les projets en cours et faire passer un certain nombre de requêtes. La voix du corps médical aura un poids particulier dans ce processus, car la FMH a su se profiler durablement comme un leader important de la politique de santé - un leader qui, rappelons-le, propose des modèles concrets qui font leurs preuves dans la pratique.

A la fin du mois, la FMH présentera à la Commission de la sécurité sociale et de la santé publique du Conseil des Etats des propositions sur la réglementation de l'après-clause du besoin et sur la gestion du système de santé. Ces propositions se fondent notamment sur des modèles d'ores et déjà appliqués avec succès par divers cantons et qui peuvent être adaptés et repris dans toute la Suisse, et aussi sur des thèses que nous avons préparées depuis plusieurs mois.

Dr Jacques de Haller, président de la FMH 